\title{
Effectiveness of Hierarchical Softmax in Large Scale Classification Tasks
}

\author{
Abdul Arfat Mohammed ${ }^{1}$ and Venkatesh Umaashankar ${ }^{2}$
}

\begin{abstract}
Typically, Softmax is used in the final layer of a neural network to get a probability distribution for output classes. But the main problem with Softmax is that it is computationally expensive for large scale data sets with large number of possible outputs. To approximate class probability efficiently on such large scale data sets we can use Hierarchical Softmax. LSHTC datasets were used to study the performance of the Hierarchical Softmax. LSHTC datasets have large number of categories. In this paper we evaluate and report the performance of normal Softmax Vs Hierarchical Softmax on LSHTC datasets. This evaluation used macro f1 score as a performance measure. The observation was that the performance of Hierarchical Softmax degrades as the number of classes increase.
\end{abstract}

Keywords - Natural Language Processing, LSHTC, Fasttext, Hierarchical Softmax

\section{INTRODUCTION}

Classification is the problem of finding to which of a set of labels a new instance belongs, on the basis of a training data set containing instances whose label membership is already known. Supervised in the Figure 1 refers to the fact that the instances of training set have their label already known.

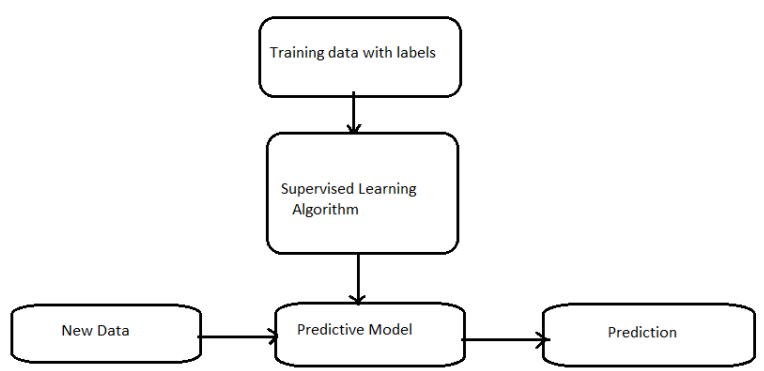

Fig. 1. Classification Block Diagram

A simple example would be like classifying fruits to a type based on past fruits dimension data. Suppose, say we want to find if a fruit is apple (or) banana based on the height of the fruit. And assume our previous data suggests that fruits that were apples had their height in range of $9-11 \mathrm{~cm}$ and that of banana was in range $13-20 \mathrm{~cm}$. Learning on above data is supervised because fruits that had height in range of $9-11 \mathrm{~cm}$ were apples and between $13-20 \mathrm{~cm}$ were bananas. Hence, our data is labelled. Our learning algorithm could be as simple as to see the new fruits height and then

\footnotetext{
${ }^{1}$ Abdul Arfat Mohammed - Intern at Ericsson R\&D, Chennai. E-mail : arfat999@gmail.com

${ }^{2}$ Venkatesh Umaashankar - Researcher at Ericsson R\&D, Chennai. E-mail : venkatesh.u@ericsson.com
}

check under which fruits height range it falls. So, using the above learning algorithm on our training data (with labels) we make a predictive model which can be used to classify new instances. Suppose, say our new fruit (new data) which is to be classified has a height of $14 \mathrm{~cm}$, we simply classify that as a banana (prediction).

Loss Function, also known as objective function is a evaluation measure of the model, typically lower the loss value better the model is . There are variety of loss functions such as cross entropy, hinge, mean squared error etc. Each loss function has its own pros and cons. The chosen loss function not only determines the model performance but also the run time and complexity. Activation functions typically take some set of inputs and map them into a non-linear space. Activation functions can also give us a probability distribution (i.e., the sum of probabilities of all nodes in the final layer is 1) for the final layer in a neural network. Some examples for activation functions are Softmax, Sigmoid, Tanh etc. Generally, In deep learning Softmax is used as an activation function on the final layer to return class probabilities for prediction purposes.

Text Classification is the task of assigning predefined categories to free-text documents. An example would be like classifying whether an email is spam or not. Similar to the above fruit classification we can train a supervised learning algorithm on a corpus of labelled emails (emails which are already categorized as Spam or not Spam) to make a prediction model which then can be used to predict new emails as they arrive either as Spam or not Spam. In Multi-class classification there are more than two categories available and the new instance can belong to only one of those categories. An example would be like classifying whether a fruit is an apple (or) a mango (or) a banana. In Multi-label classification an instance can belong to more than one category at the same time. An example would be like a document may be about politics, sports and religion at the same time.

In Large Scale Classification Tasks, we try to classify million (or) more instances into thousands (or) more categories which may be both multi-class and multi-label in nature. Example: Classifying the category of a Wikipedia document could be both multi-class and multi-label task.

In this paper, our main contributions are, (1) We prepare custom datasets with various distinct category sizes $n(=10,100,1000,10000)$ from LSHTC data sets[1] as explained in the Section III. (2) We trained Fasttext models to compare the performance of Hierarchical Softmax Vs Softmax in on these data sets using macro f1-score as an evaluation metric. (3) We report our observations and 
findings in the Section. IV

\section{LARGE SCALE ClASSIFICATION}

Large Scale Classification, typically involves dealing with millions of documents and thousands of categories. One such task is Large Scale Hierarchical Text Classification (LSHTC). In this paper, we used the data set corresponding to 4th edition of the Large Scale Hierarchical Text Classification (LSHTC) Challenge [2] for evaluating the performance of using Hierarchical Softmax as activation function instead of plain Softmax. The LSHTC Challenge is a hierarchical text classification competition, using very large datasets. The challenge is based on a large dataset created from Wikipedia. The dataset is multi-class, multi-label and hierarchical.

FastText [3] is an open-source, free, lightweight library that allows users to learn text representations and text classifiers. Also, FastText provides an implementation of Hierarchical Softmax based activation function for text classification purposes [4]. We used implementation of FastText for conducting the experiments in this paper.

\section{A. Softmax}

Softmax [5] function takes an $N$-dimensional vector of arbitrary real values and produces another $N$-dimensional vector with real values in the range $(0,1)$ that add up to 1 , i.e., Softmax turns the $N$-dimensional vector into a probability distribution which can be used for prediction purposes.

Mathematical formula:

$$
S_{i}=\frac{e^{a_{i}}}{\sum_{j=1}^{N} e^{a_{j}}}
$$

here, $S_{i}$ is the Softmax output for $i^{t h}$ value in our input vector of size $N$. Our Input vector is $\left[a_{1}, a_{2}, \ldots \ldots a_{N}\right]$.

$S_{i}$ is always positive i.e., $S_{i}>0$ because of exponents. As the numerator appears in the denominator summed up with some other positive numbers, $S_{i}<1$. Hence, this property enables us to derive a probability distribution for the classes in a classification. Typically, the Softmax is used along with the cross-entropy loss function in a neural network based classifier.

$$
\operatorname{Softmax}([1,2,3,4])=[0.03,0.08,0.24,0.64]
$$

Equation 2 shows an example of applying Softmax function on a four element input vector. The order of elements by relative size is preserved, and they add up to 1 . Intuitively, the Softmax activation function is a soft version of the maximum function.

For large numbers (positive or negative), computing Softmax may cause numeric instability due to the exponentiation. To handle this we can normalize the input vector. For detailed description on how Softmax is used in learning vector representation for words, refer to continuous bag-ofword model (CBOW) introduced in [6] and skip-gram model introduced in [7]. For further details on how the parameter learning takes place in word2vec, refer [8].

A key problem in using Softmax for Large Scale Classification is that computation becomes expensive because of the normalizing sum in the denominator term of the Equation 1. This not only affects the forward pass, but also slows down the back propagation. To solve this problem, an intuition is to limit the number of output vectors that must be updated per training instance. One way to avoid this problem is to use Hierarchical Softmax.

\section{B. Hierarchical Softmax}

Hierarchical Softmax is an efficient way of computing Softmax [9]. Binary trees are used to represent all categories in the output dictionary $(V)$ in this model. The leaf units of this binary tree represent the categories in the output dictionary $(V)$. For each leaf unit we have a unique path from the root and the probability of a category is estimated using this path. As it is a binary tree it is obvious that there are $(V-1)$ intermediate nodes. Here, the inner nodes represent internal parameters (probability mass). Each intermediate node explicitly represents the relative probabilities of its child nodes. The idea behind decomposing the output layer to a binary tree was to reduce the complexity to obtain probability distribution from $O(V)$ to $O(\log (V))$. Below Figure 2 is an example of Hierarchical binary tree (figure is from [8]):

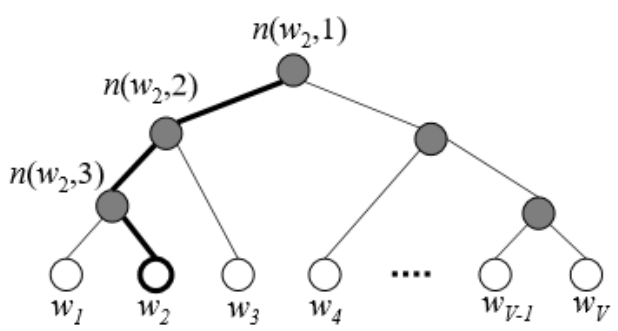

Fig. 2. An example binary tree for the Hierarchical Softmax model

The leaf nodes represent the categories and the inner nodes represent probability mass. The highlighted nodes and edges make a path from root to the above example leaf node $w_{2}$. Here, length of the path $L\left(w_{2}\right)=4$. $n(w, j)$ means the $j^{\text {th }}$ node on the path from root to a leaf node $w$. In Hierarchical Softmax model, each of the $(V-1)$ intermediate node has an output vector $v_{n(w, j)}^{\prime}$ instead of output vector representation for words. And the probability of a category $w$ being the output class will be as follows:

$p\left(w=w_{O}\right)=\prod_{j=1}^{L(w)-1} \sigma\left(\llbracket n(w, j+1)=\operatorname{ch}(n(w, j)) \rrbracket \cdot v_{n(w, j)}^{\prime T} h\right)$

here, $w_{O}$ is the actual output category. $\sigma$ is the sigmoid function. $\llbracket x \rrbracket$ : is a special function defined as:

$$
\llbracket x \rrbracket= \begin{cases}1 & \text { if } x \text { is true } \\ -1 & \text { otherwise }\end{cases}
$$


$h$ is the output of the hidden layer. $v_{n(w, j)}^{\prime}$ is the vector representation of the intermediate node $n(w, j)$ and $\operatorname{ch}(n(w, j))$ is the left child of $n(w, j)$.

Probabilities at each intermediate node in the path from root to the output category are required to compute the probability of the output category. To achieve this, at each intermediate node we must assign the probabilities for going right and going left.

We define the probability of going left at an intermediate node $n$ as follows:

$$
p(n, l e f t)=\sigma\left(v_{n}^{\prime T} \cdot h\right)
$$

And the probability of going right at the same node $n$ will obviously be:

$$
p(n, r i g h t)=1-\sigma\left(v_{n}^{\prime T} \cdot h\right)=\sigma\left(-v_{n}^{\prime T} \cdot h\right)
$$

We can calculate the probability for the category $w_{2}$ in the Figure 2 as follows:

$$
\begin{aligned}
p\left(w_{2}=w_{O}\right)= & p\left(n\left(w_{2}, 1\right), \text { left }\right) \cdot p\left(n\left(w_{2}, 2\right), \text { left }\right) \\
& \cdot p\left(n\left(w_{2}, 3\right), \text { right }\right) \\
= & \sigma\left(v_{n\left(w_{2}, 1\right)}^{\prime T} h\right) \cdot \sigma\left(v_{n\left(w_{2}, 2\right)}^{\prime T} h\right) \\
& \cdot \sigma\left(-v_{n\left(w_{2}, 3\right)}^{\prime T} h\right)
\end{aligned}
$$

For the detailed description on how the vector representations for the inner nodes are learned, refer [8].

We can also check that sum of calculated probabilities for all the words in the vocabulary add up to 1 , which is the intuition for using Softmax in the first place, but Hierarchical Softmax does this in a faster manner.

$$
\sum_{i=1}^{V} p\left(w_{i}=w_{O}\right)=1
$$

Hierarchical Softmax is a well-defined multinomial distribution among all output categories. This implies that the cost for computing the loss function and its gradient will be proportional to the number of nodes $(V)$ in the intermediate path between root node and the output node, which on average is no greater than $\log (V)$. The performance also depends on the Hierarchical tree structure used, having said that binary Huffman tree [6] [4] is expected to optimize tree for faster training.

\section{Evaluation}

LSHTC used macro f1 score as the performance metric for the evaluation criterion. To make our study comparable to their evaluation, we used the same macro $\mathrm{f} 1$ score as our performance metric. The description of the macro f1 score is detailed in the method subsection III-B.

\section{EXPERIMENTAL SETUP}

As mentioned in previous sections, we used the labeled data available in LSHTC for conducting the experiments in this paper. The entire process of creating custom data sets for top $n$ labels where, $n(=10,100,1000,10000)$ is described in method subsection III-B below. So, for four different values of $n$, four separate data sets were created from the LSHTC labeled data set. The specifications of the machine used for this experiment are as follows:

- CPU : Intel Xeon Processor E5-2650 v4 30M Cache, $2.20 \mathrm{GHz}, 12$ Cores, 24 Threads

- RAM : $250 \mathrm{~GB}$

- OS : CentOS 7

\section{A. Dataset}

The LSHTC [1] labeled data was in preprocessed form. This LSHTC is a multi-class and multi-label problem. This data set has 3,25, 056 categories to be exact and 23,65, 437 instances of labeled data is available. The format of each data file follows the libSVM format. Each line corresponds to a sparse document vector and has the following format:

label, label, label...feat : value...feat : value

label is an integer and corresponds to the category to which the document vector belongs. Each document vector may belong to more than one category. The pair feat : value corresponds to a non-zero feature with index feat and value value. feat is an integer representing a term and value is a double that corresponds to the weight ( $t f)$ of the term in the document. For example:

$$
545,328: 118: 2
$$

corresponds to a document vector whose features are all zeros except feature number 8 (with value 1) and feature number 18 (with value 2). This document vector belongs to categories 545 and 32 . Each feature number is associated to a stemmed word.

\section{B. Method}

We used Fasttext for the text classification process with the following hyper parameters:

\begin{tabular}{||cc||}
\hline Hyper Parameter Name & Hyper Parameter Value \\
\hline \hline dim (dimension for word) & 200 \\
epoch & 100 \\
lr (learning rate) & 0.25 \\
loss (loss function) & $\mathrm{hs}$ \\
\hline
\end{tabular}

TABLE I

HyPer PARAMETER VALUES USED FOR FASTTEXT

here, hs is Hierarchical Softmax.

The hierarchy file provided in LSHTC was not used. The labeled data available in LSHTC data set was split into $70 \%$ for training and $30 \%$ for testing (data was shuffled before splitting). The macro f1 score was used as performance measure. The evaluation was done for top $n$ labels each time $(n=10,100,1000,10000)$. The top $n$ signifies the top $n$ labels occurring in the LSHTC training data.

By default FastText predicts one label per sample. But, we can predict more labels per sample by giving a number as an attribute to FastText's predict function. 
As LSHTC is a multi class and multi label problem, we had to decide how many labels to predict for each sample. For this we calculated the average number of labels per doc from the LSHTC training file for each $n$. So, the evaluation is done for $n$ values of 10,100,1000,10000 by following the below steps:

1. Find the top $n$ labels occurring in the LSHTC labeled data.

2. Based on the above top $n$ labels, create a new file which has samples for only those classes from the LSHTC labeled data. If a doc has a label from top $n$ labels and other labels not from top $n$ labels then, instead of discarding the doc, only the labels which are not in top $n$ are discarded and we keep the doc to get as many number of samples for the training process. Here, we strip the term frequencies from the instances and prepend feat with ' $w$ ' to treat it as a word (as discussed in data set sub section III-A) to prepare the input data in FastText format. For example, consider

$$
545,328: 118: 2
$$

we will format this instance as follows:

$$
\text { __label__545__label__32 w8 w18 }
$$

3. After creating the new data, we find the number of average labels available for each doc and use the rounded number of that average as an argument for predict method of FastText, say it is $x$.

4. The new data is then shuffled and split into $70 \%$ train set and $30 \%$ test set.

5. Now we use FastText to train on $70 \%$ of the data using the hyper parameters shown in Table 1

6 . We then predict $x$ labels per doc ( $x$ is from step 3 ) using fasttext.

7. For evaluation we use macro f1 score $(M a F)$ as follows:

$$
M a F=\frac{2 * M a P * M a R}{M a P+M a R}
$$

Here, $M a P$ is macro precision and $M a R$ is macro recall.

$$
\begin{aligned}
M a P & =\frac{\sum_{i=1}^{|C|} \frac{t p_{c_{i}}}{t p_{c_{i}}+f p_{c_{i}}}}{|C|} \\
M a R & =\frac{\sum_{i=1}^{|C|} \frac{t p_{c_{i}}}{t p_{c_{i}}+f n_{c_{i}}}}{|C|}
\end{aligned}
$$

where, $C$ is set of classes, $t p_{c_{i}}, f n_{c_{i}}$ and $f p_{c_{i}}$ are the true positives, false negatives and false positives respectively for class $c_{i}$. The above process is repeated for each value of $n$.

\section{RESUlTS}

The following tables show the results obtained for various top $n$ labels in LSHTC training data. All macro precision, macro recall, macro f1 scores in the Tables III and IV are rounded to two decimal places.

\begin{tabular}{||ccc||}
\hline Top $n$ labels & avg labels per doc & labels predicted per doc \\
\hline \hline 10 & 1.2 & 1 \\
100 & 1.4 & 1 \\
1000 & 1.9 & 2 \\
10000 & 2.4 & 2 \\
\hline
\end{tabular}

TABLE II

RESULTS OBTAINED FOR VARIOUS TOP $n$ LABELS DATA SET

\begin{tabular}{||cccc||}
\hline Top $n$ labels & macro precision & macro recall & macro f1 \\
\hline \hline 10 & 0.77 & 0.45 & 0.54 \\
100 & 0.51 & 0.30 & 0.35 \\
1000 & 0.34 & 0.34 & 0.32 \\
10000 & 0.25 & 0.22 & 0.21 \\
\hline
\end{tabular}

TABLE III

SCORES OBTAINED FOR VARIOUS TOP $n$ LABELS USING Hierarchical Softmax

\begin{tabular}{||cccc||}
\hline Top $n$ labels & macro precision & macro recall & macro f1 \\
\hline \hline 10 & 0.82 & 0.48 & 0.58 \\
100 & 0.69 & 0.40 & 0.47 \\
1000 & 0.55 & 0.46 & 0.47 \\
10000 & 0.49 & 0.37 & 0.38 \\
\hline
\end{tabular}

TABLE IV

SCORES OBTAINED FOR VARIOUS TOP $n$ LABELS USING Softmax

\begin{tabular}{||ccc||}
\hline Top $n$ labels & Hierarchical Softmax & Softmax \\
\hline \hline 10 & $1 \mathrm{~min}$ & $1 \mathrm{~min}$ \\
100 & $1 \mathrm{~min}$ & $5 \mathrm{~min}$ \\
1000 & $2 \mathrm{~min}$ & $51 \mathrm{~min}$ \\
10000 & $5 \mathrm{~min}$ & $15 \mathrm{Hr} 11 \mathrm{~min}$ \\
\hline
\end{tabular}

TABLE V

TRAINING TIMES FOR MODELS WHEN Hierarchical Softmax AND Softmax ARE USED

Based on top $n(=10,100,1000,10000)$ labels, data sets for each $n$ are created separately as mentioned in the method sub section III-B. Average labels per doc are calculated from created data set (different for each of top $n$ labels). Labels predicted per doc is the number of labels we are predicting (using fasttext predict option) for each doc in test set of our created data set (different for each of top $n$ labels). The macro precision, macro recall, macro $\mathrm{fl}$ scores are calculated as described in the method sub section III-B The Figure 3 below, shows how the macro f1 score (for top $n$ labels) varies when Hierarchical Softmax and Softmax are used on the final layer of a neural network. We can also see that the macro $\mathrm{f} 1$ score decreases as the number of labels increase. 


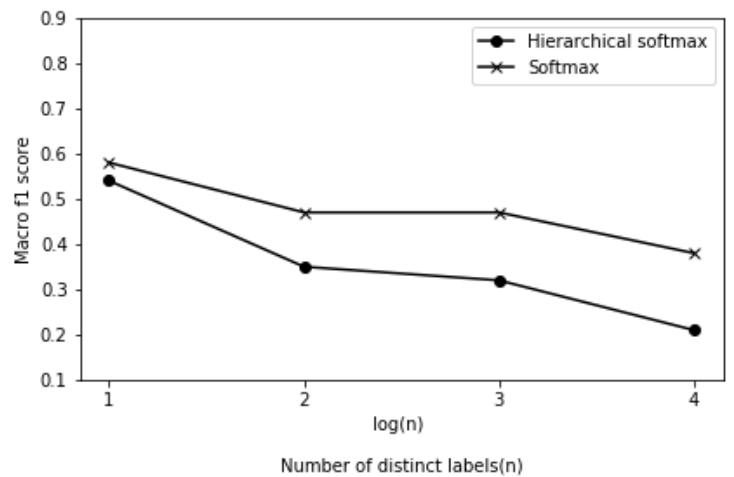

Fig. 3. Comparison of macro f1 scores when Hierarchical Softmax and Softmax are used

In the Figure 3 above, we used the $\log _{10}$ scale for the top $n$ classes because as the size of the labels grows the fall in the macro f1 score was increasing and it was not intuitive enough to view on scale where $n$ ranges from 10 to 10000 , hence the graph was plotted with $\log _{10}(n)$ on the $x$-axis and with the macro f1 score on the $y$-axis.

From Tables III, IV and V. It is evident that Hierarchical Softmax indeed improves training speed when compared to Softmax, but at the cost of being less accurate.

\section{CONCLUSION}

In this paper, we compared the performance of Hierarchical Softmax and Softmax in large scale classification tasks on LSHTC data. Our conclusions are as follows: (1) Softmax performs better when compared to Hierarchical Softmax in large scale classification tasks. (2) Hierarchical Softmax is indeed faster when compared to Softmax in training a model.

In our future work, we will expand our study in the following areas: (1) Evaluate using a data set that is in raw text form and not in pre-processed form. (2) Use better model to find the optimal number of labels to predict per doc instead of simple averaging based approach. (3) Combine FastText embeddings with a Recurrent Neural Network (RNN) to treat the hierarchical classification as a sequence prediction problem.

\section{REFERENCES}

[1] I. Partalas, M.-R. Amini, I. Androutsopoulos, T. Artires, N. Baskiotis, P. Gallinari, E. Gaussier, A. Kosmopoulos, and G. Paliouras, "Large scale hierarchical text classification - kaggle," Jan 2014. [Online]. Available: https://www.kaggle.com/c/lshtc

[2] I. Partalas, A. Kosmopoulos, N. Baskiotis, T. Artières, G. Paliouras, É. Gaussier, I. Androutsopoulos, M. Amini, and P. Gallinari, "LSHTC: A benchmark for large-scale text classification," CoRR, vol. abs/1503.08581, 2015.

[3] "Fasttext, a library for efficient text classification and representation learning." [Online]. Available: https://fasttext.cc/

[4] A. Joulin, E. Grave, P. Bojanowski, and T. Mikolov, "Bag of tricks for efficient text classification," arXiv preprint arXiv:1607.01759, 2016.

[5] E. Bendersky, "The softmax function and its derivative," Oct 2016. [Online]. Available: https://eli.thegreenplace.net/2016/ the-softmax-function-and-its-derivative/
[6] T. Mikolov, K. Chen, G. Corrado, and J. Dean, "Efficient estimation of word representations in vector space," arXiv preprint arXiv:1301.3781, 2013.

[7] T. Mikolov, I. Sutskever, K. Chen, G. S. Corrado, and J. Dean, "Distributed representations of words and phrases and their compositionality," in Advances in neural information processing systems, 2013, pp. 3111-3119.

[8] X. Rong, "word2vec parameter learning explained," arXiv preprint arXiv:1411.2738, 2014.

[9] F. Morin and Y. Bengio, "Hierarchical probabilistic neural network language model." in Aistats, vol. 5. Citeseer, 2005, pp. 246-252. 